\title{
Low-temperature sterilization of wrapped materials using flexible sheet-type dielectric barrier discharge
}

\author{
Hiroyuki Eto, Yoshihito Ono, Akihisa Ogino, and Masaaki Nagatsu ${ }^{a}$ \\ Graduate School of Science and Engineering, Shizuoka University, 3-5-1 Johoku, \\ Hamamatsu 432-8561, Japan
}

(Received 6 September 2008; accepted 11 November 2008; published online 1 December 2008)

\begin{abstract}
A flexible sheet-type dielectric barrier discharge (DBD) was studied for the low-temperature sterilization of medical instruments wrapped with Tyvek packaging. Sterilization experiments using Geobacillus stearothermophilus spores with a population of $10^{6}$ were carried out with various mixtures of nitrogen and oxygen. We confirmed the inactivation of spores after $4.5 \mathrm{~min}$ of DBD irradiation at a temperature of $28.4{ }^{\circ} \mathrm{C}$ and relative humidity of $64.4 \%$. The main sterilizing factors of this method are the ozone and UV emissions generated by DBD in dry air and synergistic $\mathrm{OH}$ radicals generated by DBD in moist air. (C) 2008 American Institute of Physics.
\end{abstract}

[DOI: $10.1063 / 1.3039808]$

Atmospheric pressure discharges have been widely investigated in recent years in order to develop lowtemperature material processing techniques for a variety of industrial applications, such as surface treatment, thin-film deposition, detoxification of gaseous pollution, and sterilization. One of the advantages of atmospheric-pressure plasma is that it does not require a costly pump system. Therefore, prompt material processing without using batch systems can be realized. Among the various applications, plasma sterilization techniques in the medical field and food industry have recently been proposed.

To date, substantial research has been carried out to investigate sterilization techniques using low-pressure plasma $^{1-7}$ and atmospheric pressure plasma. ${ }^{8-14}$ The roles of the various plasma agents in the inactivation of bacteria were studied in detail by Lu et al. ${ }^{15}$ using a plasma jet in the atmospheric pressure. Most of these researches, however, have dealt with the direct surface treatment of microorganisms. With regard to practical use in medical facilities such as hospitals or medical clinics, however, sterilization of various kinds of medical instruments wrapped by sterilization bags is mandatory. Therefore, it is important to develop plasma sterilization techniques to inactivate viruses and other microorganisms inside specific plastic packaging, such as Tyvek packaging (product of DuPont Co.).

In the present study, we thus aimed at developing a lowtemperature, atmospheric sterilization technique for treating medical instruments wrapped with Tyvek packaging. We employed a flexible sheet-type dielectric barrier discharge (DBD) plasma to fit any shapes of Tyvek packaging and applied it to the inner sterilization of wrapped biological indicators (BIs). We demonstrated that Geobacillus stearothermophilus spores having a population of $10^{6}$ spores inside the packages were successfully inactivated after $4.5 \mathrm{~min}$ of DBD plasma irradiation. In order to investigate the sterilization mechanisms, we then carried out sterilization experiments by varying the gas mixture ratio of $\mathrm{N}_{2}$ and $\mathrm{O}_{2}$. Based on the present study, we believe that the main sterilizing factors are ozone and UV emissions generated by DBD in dry air, and

a) Tel.: +81-53-478-1081. Electronic mail: tmnagat@ipc.shizuoka.ac.jp. particularly in moist air, the generated $\mathrm{OH}$ radicals expedite the sterilization properties.

In order to investigate the internal sterilization of Tyvekwrapped medical instruments, we proposed the use of a flexible sheet-type DBD, as shown in Fig. 1. Figure 1 shows the structure of the flexible sheet-type DBD with a width of 40 $\mathrm{mm}$ and a length of $40 \mathrm{~mm}$ and an aluminum tape rectangle form with a width $50 \mathrm{~mm}$ and a length $55 \mathrm{~mm}$. Aluminum tape and stainless steel mesh were used as electrodes, and these sandwiched a resin sheet acting as a dielectric layer. High-voltage ac, typically around $\pm 2.5 \mathrm{kV}$ at a frequency of $5 \mathrm{kHz}$, was applied between the two electrodes to generate the DBD over the mesh electrode. The BI Geobacillus stearothermophilus spores, ${ }^{16}$ with a population of $3.0 \times 10^{6}$, were pasted to a bowl-shaped stainless steel disk, which was wrapped in a $130 \mathrm{~mm}^{2}$ Tyvek package consisting of a nonwoven fabric Tyvek sheet and a transparent polyethylene film. The nonwoven fabric sheet is perforated and permeable to air. The sheet type of DBD electrodes were directly attached to the nonwoven fabric side of the Tyvek package.

Here, we used two methods to judge the sterilization of BIs. First, we confirmed whether the spores are perfectly inactivated by examining the color of tryptic soy broth solution in a culture tube after incubating the samples for 7 days or longer at $55-60{ }^{\circ} \mathrm{C}$. If the spores were still viable, the color changed from purple to yellow. Second, we confirmed by analyzing the colony forming units using a conventional colony counting method.

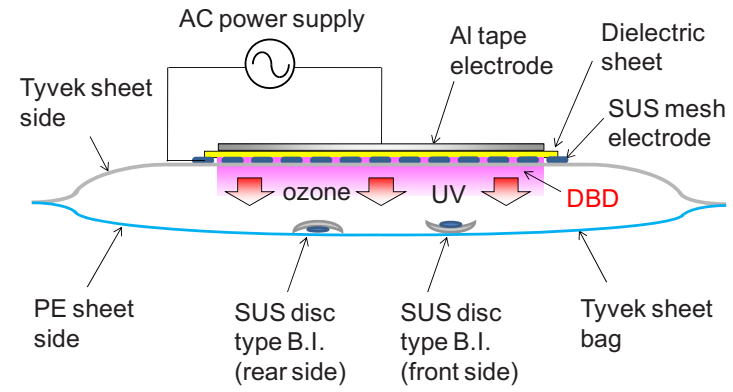

FIG. 1. (Color online) Schematic view of flexible sheet-type DBD. 
(a)

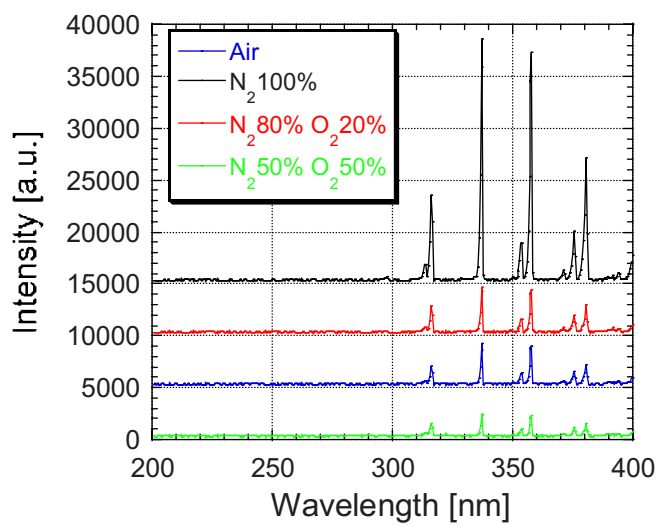

(b)

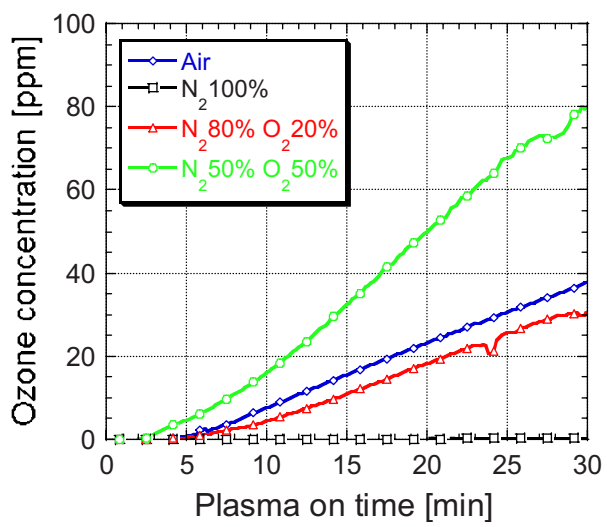

FIG. 2. (Color online) (a) Emission spectrum and (b) ozone concentrations generated by DBD under different ratios of $\mathrm{N}_{2}$ and $\mathrm{O}_{2}$ : (i) air with a relative humidity of $64.4 \%$, (ii) $\mathrm{N}_{2} \quad 100 \%$, (iii) $\mathrm{N}_{2} \quad 80 \%$ and $\mathrm{O}_{2} 20 \%$ (simulated dry air), and (iv) $\mathrm{N}_{2} 50 \%$ and $\mathrm{O}_{2} 50 \%$.

In order to clarify the present sterilization mechanism, we also carried out optical emission spectrometry (OES) and ozone concentration measurements under different gas conditions by changing the ratio of nitrogen and oxygen gas in the experimental stainless steel chamber. OES measurements were performed using a UV-visible spectrometer (Acton, SpectraPro 2300i) having a wavelength range of 200-800 $\mathrm{nm}$. Measurement of ozone concentration was performed using an ozone analyzer (Eco Sensors, Ozone Analyzer UV100). These OES and ozone concentration measurements were carried out in a cylindrical stainless steel chamber having a diameter of $250 \mathrm{~mm}$ and a length of $500 \mathrm{~mm}$, in which the sandwich-type DBD electrodes were installed and DBD plasma was ignited by applying an ac voltage of $\pm 2.5 \mathrm{kV}$ at a frequency of $5 \mathrm{kHz}$. The typical waveforms of the applied voltage and flowing current of the sheet DBD electrodes were similar to those obtained for conventional plane-type DBD electrodes; spiky current peaks were only observed in the rising phase of positive polarity and the falling phase of negative phase of the applied voltage. DBD occurred at the outside edge of the stainless mesh and then became a uniform discharge over the mesh electrode.

In the sterilization experiment, we inserted two BIs pasted on stainless steel disks into the Tyvek package. One side faced the DBD and the other did not to compare a difference in sterilization property between them. We first tested the effects of temperature using the sheet DBD. Temperature during DBD plasma irradiation was measured using thermosensitive sheets where the thermosensitive marks for the respective temperatures changed color from white to black (a)
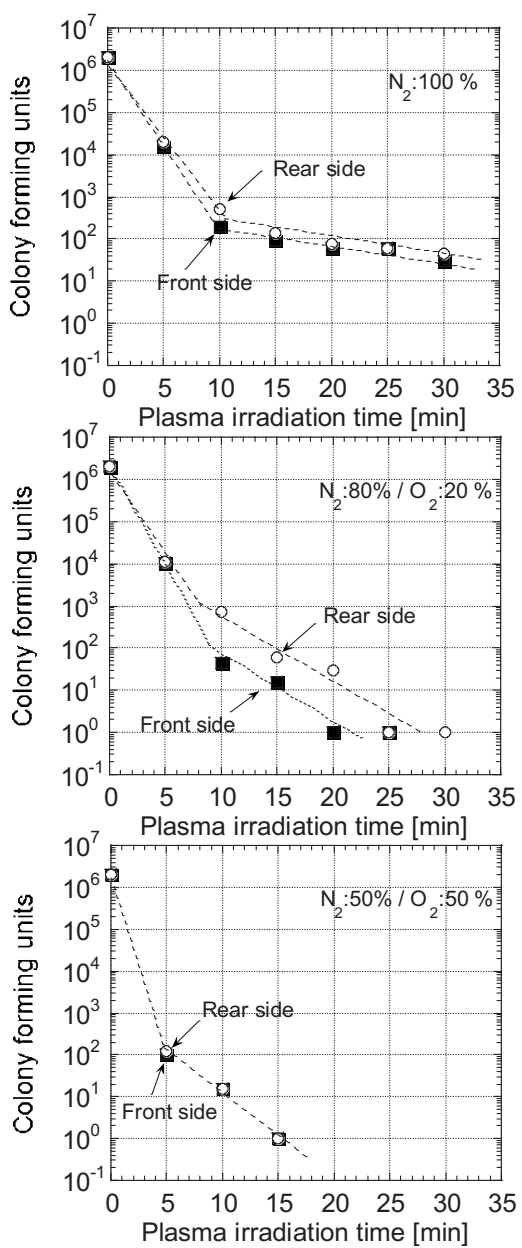

FIG. 3. Results of colony count measurement of BIs under different ratios of $\mathrm{N}_{2}$ and $\mathrm{O}_{2}$ : (a) $\mathrm{N}_{2} 100 \%$, (b) $\mathrm{N}_{2} 80 \%$ and $\mathrm{O}_{2} \quad 20 \%$, and (c) $\mathrm{N}_{2} 50 \%$ and $\mathrm{O}_{2} 50 \%$.

when those specified temperatures were surpassed. The plasma irradiation time varied up to 20 min under the applied voltage of $\pm 2.5 \mathrm{kV}$ at a frequency of $5 \mathrm{kHz}$. After a $20 \mathrm{~min}$ plasma irradiation, the temperature of the Tyvek package increased from room temperature $\left(15^{\circ} \mathrm{C}\right)$ to roughly $50{ }^{\circ} \mathrm{C}$. Thus, we confirmed that the Tyvek sheet was not damaged by DBD plasma.

Figure 2(a) shows the OES spectra of discharge under different gas compositions. The emission intensities of the $\mathrm{N}_{2}$ second positive system are dominant in the wavelength range from 200 to $400 \mathrm{~nm}$ and increased with $\mathrm{N}_{2}$ gas addition. Figure 2(b) shows the temporal behavior of ozone concentration for different gas compositions. The ozone concentration increased with the oxygen gas ratio. In order to clarify the role of ozone in the process of sterilization, the emission of the ozone at $313.74,322.15$, or $322.72 \mathrm{~nm}$ (Refs. 17 and 18) itself rather than that of atomic oxygen might be analyzed. It is considered that the present DBD plasma was generated only near the sheetlike DBD electrode and the ozone was produced via a reaction between oxygen atoms generated by DBD plasma and oxygen molecules in the surrounding volume far from the plasma region. Therefore, we may consider that the ozone itself is not directly excited by DBD plasma. When we increased the oxygen content to $\mathrm{N}_{2} 50 \% / \mathrm{O}_{2} 50 \%$, we observed a weak peak near the wavelengths of 322.72 and $322.15 \mathrm{~nm}$. As for the emission at $313.74 \mathrm{~nm}$, there exists a strong peak of $\mathrm{N}_{2}$ emission at 


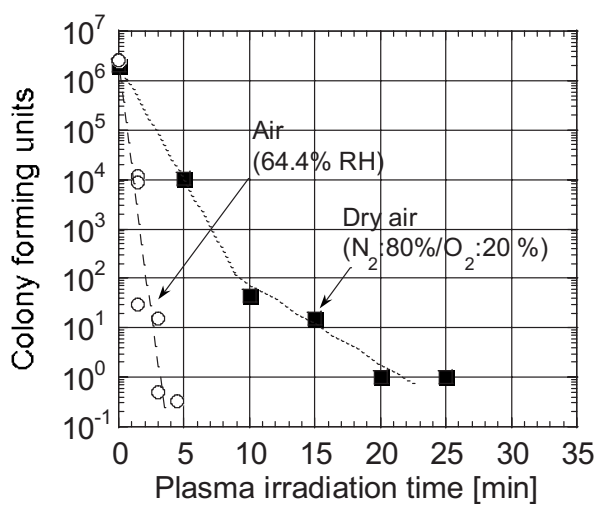

FIG. 4. Results of colony count measurement of BIs under atmospheric conditions at room temperature $\left(28.4{ }^{\circ} \mathrm{C}\right)$ and a relative humidity of $64.4 \%$. (a) Spores deposited on front side of sheet electrode and (b) spores deposited on the back side.

$313.60 \mathrm{~nm}$, so we could not separate the ozone emission. In the present experimental condition, excitation efficiency of ozone emission is not enough to discuss the relation between the ozone concentration and its UV emission.

We examined the effects of gas mixture conditions on sterilization properties by varying the gas ratio of $\mathrm{N}_{2}$ and $\mathrm{O}_{2}$. Figure 3 shows the experimental results of colony count measurements in the samples treated under the different gas mixtures: (a) $\mathrm{N}_{2}$ 100\%, (b) $\mathrm{N}_{2} 80 \%$ and $\mathrm{O}_{2} 20 \%$, and (c) $\mathrm{N}_{2} 50 \%$ and $\mathrm{O}_{2} 50 \%$. The spores were clearly inactivated more rapidly with higher $\mathrm{O}_{2}$ gas contents.

In the case of a $100 \% \mathrm{~N}_{2}$ atmosphere, the surviving spores decreased at two different rates with plasma irradiation time, as shown in Fig. 3(a). Here, the UV emissions ranging from 300 to $400 \mathrm{~nm}$ due to the $\mathrm{N}_{2}$ second-positive system are a possible sterilizing factor. When compared with the rear side BI, the front side BI showed a slightly faster decay. This is because the UV emissions from $\mathrm{N}_{2}$ plasma directly inactivated the spores on the front side BI. However, it was also noted that the rear side BI was inactivated. We believe that the UV emissions generated by DBD plasma were reflected to the spores via the Tyvek package or the stainless steel chamber wall.

As $\mathrm{O}_{2}$ ratio increased, we observed more rapid inactivation due to ozone production, as shown in Figs. 3(b) and 3(c). In Fig. 3(c), the survival curves for front side BI and rear side BI are the same, which indicates that ozone is the dominant sterilizing factor. Furthermore, we observed that the spores were inactivated within $5 \mathrm{~min}$ in actual air with humidity of $64.4 \%$, as shown in Fig. 4. This shows that the water vapor may also play an important role in sterilization. In the present experiment, however, we could not observe the $\mathrm{OH}$ line at $308.9 \mathrm{~nm}$ in the case of wet air [see Fig. 2(a)]. At present, we consider that the $\mathrm{OH}$ species might be produced not by plasma discharge directly but by the chemical reaction between ozone and water vapor in the surrounding region far from the DBD plasma localized near electrode. To confirm the existence of $\mathrm{OH}$ radicals, the further study will be needed.

Spores treated with DBD plasma were analyzed under a scanning electron microscope (SEM). ${ }^{13}$ The SEM images of the spores treated with DBD plasma under humid air confirmed that the shapes of spores were changed by the lytic action of DBD plasma. It is likely that the $\mathrm{OH}$ radicals produced via direct chemical reactions in the DBD in humid air or a UV-induced chemical reaction between water vapor and ozone generated by DBD play a role. In the present experiment, we also consider that $\mathrm{OH}$ radicals would act as strong oxidants and breach the outer membrane of the spores.

In this study, we discuss the internal sterilization of wrapped medical instruments using sheet-type DBD plasma. We also compared the experimental sterilization results by varying the $\mathrm{N}_{2}$ and $\mathrm{O}_{2}$ ratios. $\mathrm{UV}$, ozone, and chemical reactions in water vapor were considered to contribute to the sterilizing effects, although ozone and $\mathrm{OH}$ radicals were thought to play the major role in the present experiment.

This work was partly supported by a Grant-in-Aid for Scientific Research from the Japan Society for the Promotion of Science (JSPS). The authors would also like to thank Metran Co. for their collaboration.

${ }^{1}$ M. Moisan, J. Barbeau, S. Moreau, J. Pellettier, M. Tabrizian, and L'H. Yahia, Int. J. Pharm. 226, 1 (2001).

${ }^{2}$ N. Philip, B. Saoudi, M. C. Crevier, M. Moisan, J. Barbeau, and J. Pelletier, IEEE Trans. Plasma Sci. 30, 1429 (2002).

${ }^{3}$ M. Moisan, B. Saoudi, M. C. Crevier, N. Philip, E. Fafard, J. Barbeau, and J. Pelletier, Conference Proceedings of Fifth International Workshop Microwave Discharges: Fundamentals and Applications, Greifswald, Germany, July 8-12, 2003 (unpublished), pp. 210-221.

${ }^{4}$ M. Nagatsu, F. Terashita, Y. Koide. Jpn. J. Appl. Phys., Part 2 42, L856 (2003).

${ }^{5}$ M. Nagatsu, F. Terashita, H. Nonaka, L. Xu, T. Nagata, and Y. Koide, Appl. Phys. Lett. 86, 211502 (2005).

${ }^{6}$ J. Feichtinger, A. Schulz, M. Walker, and U. Schumacher, Surf. Coat. Technol. 174, 564 (2003).

${ }^{7}$ L. Xu, H. Nonaka, H. Y. Zhou, A. Ogino, T. Nagata, Y. Koide, S. Nanko, I. Kurawaki, and M. Nagatsu, J. Phys. D 40, 803 (2007).

${ }^{8}$ M. Laroussi, Plasma Process. Polym. 2, 391 (2005).

${ }^{9}$ B. J. Park, K. Takatori, M. H. Lee, D. W. Han, Y. I. Woo, H. J. Son, J. K. Kim, K. H. Chung, S. O. Hyun, and J. C. Park, Surf. Coat. Technol. 201, 5738 (2007)

${ }^{10}$ J. H. Choi, I. Han, H. K. Baik, M. L. Lee, D. W. Han, J. C. Park, I. S. Lee, K. M. Song, and Y. S. Lim, J. Electrost. 64, 17 (2006).

${ }^{11}$ N. Ekem, T. Akan, Y. Akgun, A. Kiremitci, S. Pat, and G. Musa, Surf Coat. Technol. 201, 993 (2006).

${ }^{12}$ T. Akitsu, H. Ohkawa, M. Tsuji, H. Kimura, and M. Kogoma, Surf. Coat. Technol. 193, 29 (2005).

${ }^{13}$ H. Ohkawa, T. Akitsu, M. Tsuji, H. Kimura, M. Kogoma, and K. Fukushima, Surf. Coat. Technol. 200, 5829 (2006).

${ }^{14}$ H. Eto, Y. Ono, A. Ogino, and M. Nagatsu, Plasma Process. Polym. 5, 269 (2008).

${ }^{15}$ X. P. Lu, T. Ye, Y. G. Cao, Z. Y. Sun, Q. Xiong, Z. Y. Tang, Z. L. Xiong, J. Hu, Z. H. Jiang, and Y. Pan, J. Appl. Phys. 104, 053309 (2008).

${ }^{16}$ ATCC No. 12980.

${ }^{17}$ R. W. B. Pears and A. G. Gaydon, The Identification of Molecular Spectra, 4th ed. (Chapman \& Hall, London, 1976), p. 263.

${ }^{18}$ J. Janin Acad. Sci., Paris, C. R. 207, 145 (1938). 